to appear, AWM-Springer Series, Harmonic Analysis, Partial Differentail Equations, Complex Analysis, Banach Spaces, and Operator Theory. Celebrating Cora Sadosky's Life. Editors: S. Marcantognini, C. Pereyra, A. Stokolos and W. Urbina.

\title{
THREE OBSERVATIONS ON COMMUTATORS OF SINGULAR INTEGRAL OPERATORS WITH BMO FUNCTIONS
}

\author{
CARLOS PÉREZ AND ISRAEL P. RIVERA-RÍOS
}

Abstract. Three observations on commutators of Singular Integral Operators with BMO functions are exposed, namely

1- The already known subgaussian local decay for the commutator, namely

$$
\frac{1}{|Q|}\left|\left\{x \in Q:\left|[b, T]\left(f \chi_{Q}\right)(x)\right|>M^{2} f(x) t\right\}\right| \leq c e^{-\sqrt{c t\|b\|_{B M O}}}
$$

is sharp, since it cannot be better than subgaussian.

2. It is not possible to obtain a pointwise control of the commutator by a finite sum of sparse operators defined by $L \log L$ averages.

3. Motivated by the conjugation method for commutators, it is shown the failure of the following endpoint estimate, if $w \in A_{p} \backslash A_{1}$ then

$$
\left\|w M\left(\frac{f}{w}\right)\right\|_{L^{1}\left(\mathbb{R}^{n}\right) \rightarrow L^{1, \infty}\left(\mathbb{R}^{n}\right)}=\infty .
$$

\section{INTRODUCTION}

The purpose of this paper is to present some observations concerning commutators of singular integral operators with BMO functions. These operators were introduced by Coifman, Rochberg and Weiss in [6] as a tool to extend the classical factorization theorem for Hardy spaces in the unit circle to $\mathbb{R}^{n}$. These operators are defined by the expression

$$
T_{b} f(x)=\int_{\mathbb{R}^{n}}(b(x)-b(y)) K(x, y) f(y) d y,
$$

where $K$ is a kernel satisfying the standard Calderón-Zygmund estimates and where $b$, the "symbol" of the operator, is a locally integrable function. Of course, these are special cases of the more general commutators given by the expression

$$
T_{b}=[b, T]=M_{b} \circ T-T \circ M_{b}
$$

where $T$ is any operator and $M_{b}$ is the multiplication operator $M_{b} f=b \cdot f$.

The classical well known result from [6] establishes that $[b, T]$ is a bounded operator on $L^{p}\left(\mathbb{R}^{n}\right), 1<p<\infty$, when the symbol $b$ is a $B M O$ function. We state this result.

2010 Mathematics Subject Classification. 42B35,46E30.

The first author was supported by Severo Ochoa Excellence Programme and the Spanish Government grant MTM2014-53850-P and the second author was supported by Grant MTM2012-30748, Spanish Government. 
Theorem 1. Let $T$ be a singular integral operator and $b$ a BMO function. The commutator $T_{b}$ is bounded on $L^{p}\left(\mathbb{R}^{n}\right)$ for every $1<p<\infty$.

In the same paper it is shown that $b \in B M O$ is also a necessary condition namely, if the commutators $\left[b, R_{j}\right], j=1, \cdots, n$ of $b$ with the Riesz transforms $R_{j}$ are bounded on $L^{p}\left(\mathbb{R}^{n}\right)$ for some $p \in(1, \infty)$ and every $j \in\{1,2, \ldots, n\}$ then $b \in B M O$.

None of the different proofs of this result follows the usual scheme of the classical Calderón-Zygmund theory for proving the $L^{p}\left(\mathbb{R}^{n}\right)$ boundedness of singular integral operators $T$. Two proofs of Theorem 1 can be found in [6]. The first and main one in that paper is based on methods involving techniques similar to those used in [5] to understand the Calderón commutator. As far as we know this approach has not been so influential. However, the second proof, based on the so called conjugation method from operator theory, has been widely used. In fact, it is quite surprising that this proof was postponed to the end of the paper since it turns out to be highly interesting. Indeed, the method shows the intimate connection between these commutators and the $A_{p}$ theory of weights. Furthermore, this proof can be applied to general linear operators, not only for Singular Integral Operators. As a sample we will point out the following particular $L^{2}$ case:

Theorem 2. Suppose that $T$ is a linear operator such that

$$
T: L^{2}(w) \longrightarrow L^{2}(w)
$$

for every $w \in A_{2}$. Then for every $b \in B M O$,

$$
[b, T]: L^{2}\left(\mathbb{R}^{n}\right) \longrightarrow L^{2}\left(\mathbb{R}^{n}\right) .
$$

The method of proof can be carried out in more generality as shown in [1]. The key initial argument of the proof is that we can write $[b, T]$ as a complex integral operator using the Cauchy integral theorem as follows

$$
[b, T] f=\left.\frac{d}{d z} e^{z b} T\left(f e^{-z b}\right)\right|_{z=0}=\frac{1}{2 \pi i} \int_{|z|=\varepsilon} \frac{T_{z}(f)}{z^{2}} d z, \quad \varepsilon>0
$$

where

$$
z \rightarrow T_{z}(f):=e^{z b} T\left(\frac{f}{e^{z b}}\right) \quad z \in \mathbb{C} .
$$

This is called the "conjugation" of $T$ by $e^{z b}$ and the terminology comes most probably from group theory. Now, if $\|\cdot\|$ is a norm we can apply Minkowski inequality:

$$
\|[b, T] f\| \leq \frac{1}{2 \pi \varepsilon} \sup _{|z|=\varepsilon}\left\|T_{z}(f)\right\| \quad \varepsilon>0 .
$$

The effectiveness of the method can be checked in the modern context of weighted $L^{p}$ estimates. Indeed, the method produces very optimal bounds of the operator norm as shown in [4] (see also [15]).

This method reveals the role played by the following operation:

$$
f \rightarrow T_{w}(f):=w T\left(\frac{f}{w}\right)
$$


where $w$ is a weight which, in this context, is an $A_{p}$ weight. Indeed, this is the case by the well known key property of the BMO class, if $p>1$ and $b \in B M O$ then there is a small $\varepsilon_{0}$, such that $e^{t b} \in A_{p}$, for any real number $t$ such that $|t|<\varepsilon_{0}$. These operators were already studied by B. Muckenhoupt and R. Wheeden in the 70's and by E. Sawyer in the 80's. Some of the problems they left open were solved in [8]. A consequence of the main result of [8] is that if $w \in A_{1}$ then $T_{w}$ is of weak type $(1,1)$, namely

$$
\left\|T_{w}\right\|_{L^{1}\left(\mathbb{R}^{n}\right) \rightarrow L^{1, \infty}\left(\mathbb{R}^{n}\right)}<\infty
$$

with bound depending upon the $A_{1}$ constant of $w$. However, we will exhibit examples of weights $w \in A_{p} \backslash A_{1}$ in Section 4 for which $T_{w}$ is not of weak type $(1,1)$, namely

$$
\left\|T_{w}\right\|_{L^{1}\left(\mathbb{R}^{n}\right) \rightarrow L^{1, \infty}\left(\mathbb{R}^{n}\right)}=\infty
$$

This shows that the case $w \in A_{1}$ is specially relevant. Perhaps, this phenomenon can be explained by the fact that the conjugation method is closely attached to commutators with BMO functions which are not of weak type $(1,1)$ as observed in [25]. Indeed, the conjugation method works due to the property, already mentioned, that if $p>1$ and $b \in B M O$ then $e^{t b} \in A_{p}$ for small values of $t$. However, this property turns out to be false in the case $p=1$. The lack of the weak type $(1,1)$ property for commutators is replaced by a $L \log L$ inequality like (5) below and not better.

There is another proof of Theorem 1 based on the use of the sharp maximal function of C. Fefferman and E. Stein which has also been very influential. It seems that it was first discovered by J. O. Strömberg as mentioned by S. Janson in [17] (see also [28] pp. 417-419) The proof relies on combining the following key pointwise estimate

$$
M^{\sharp}([b, T] f) \leq c\|b\|_{B M O}\left(M_{r}(T f)+M_{s}(f)\right)
$$

where $1<r, s<\infty$ and $M_{r}(f)=M\left(|f|^{r}\right)^{1 / r}$ together with the classical Fefferman-Stein inequality:

$$
\|M(f)\|_{L^{p}} \leq c\left\|M^{\sharp}(f)\right\|_{L^{p}} .
$$

Here we use standard notation, $M$ is the Hardy-Littlewood maximal function and $M^{\sharp}$ is the sharp maximal function. The $L^{p}$ boundedness of $M$ and $T$ yields the alternative proof of Theorem 1. Proceeding in the same way we obtain the corresponding estimates for $A_{p}$ weights.

This approach was considered by S. Bloom in ([2]) extending in an interesting way Theorem 1 but only on the real line.

Theorem 3. Let $\mu, \lambda \in A_{p}$ and let $H$ be the Hilbert transform:

$$
[b, H]: L^{p}(\mu) \longrightarrow L^{p}(\lambda)
$$

where $\nu=\mu^{\frac{1}{p}} \lambda^{-\frac{1}{p}}$ if and only if

$$
\|b\|_{B M O(\nu)}=\sup _{Q} \frac{1}{\nu(Q)} \int_{Q}\left|b-b_{Q}\right|<\infty .
$$

The power of the pointwise estimate (2) is reflected in many situations, for instance in [12], where similar results were derived for commutators of strongly singular integral with symbol in the new $B M O$ class (3) (see also [13, 14] for an alternative approach based on dyadic shifts). 
However, estimate (2) is not sharp enough for many purposes and much better results can be obtained with the following variation:

$$
M_{\delta}^{\sharp}([b, T] f) \leq c\|b\|_{B M O}\left(M_{\varepsilon}(T f)+M^{2}(f)\right) \quad 0<\delta<\varepsilon<1
$$

where $M^{2}$ stands for $M \circ M$ (see [25]). Here, the key difference is that we are considering small parameters $\delta$ and $\varepsilon$. The estimate is sharp since $M^{2}$ cannot be replaced by the (pointwise) smaller operator $M$. Indeed, otherwise these commutators would be of weak type $(1,1)$ but, as we mentioned above, this is not the case [25] where it is shown that commutators satisfy the following " $L \log L$ " type estimate,

$$
w\left(\left\{x \in \mathbb{R}^{n}:|[b, T] f(x)|>\lambda\right\}\right) \leq c \int_{\mathbb{R}^{n}} \Phi\left(\frac{|f|}{\lambda}\|b\|_{B M O}\right) w d x \quad \lambda>0,
$$

where $w \in A_{1}, \Phi(t)=t \log (e+t)$ and where $c>0$ depends upon the $A_{1}$ constant. This shows that these commutators are "more singular" than Calderón-Zygmund operators. The original proof of (5) follows from the key pointwise (4) combined with a good- $\lambda$ type argument, but an alternative proof was obtained by the first author and G. Pradolini in [26] with the bonus that non $A_{\infty}$ weights can be considered. This argument is based on a variation of the classical scheme used to prove the weak type $(1,1)$ for CalderónZygmund operators. The statement of the result is the following.

Theorem 4. Let $T$ be a Calderón-Zygmund operator and $b \in B M O$. If $w$ is an arbitrary weight the following inequality holds

$$
w\left(\left\{x \in \mathbb{R}^{n}:|[b, T] f(x)|>\lambda\right\}\right) \leq C_{\varepsilon, T} \int_{\mathbb{R}^{n}} \Phi\left(\|b\|_{B M O} \frac{|f(x)|}{\lambda}\right) M_{L(\log L)^{1+\varepsilon}} w(x) d x
$$

for every $\varepsilon>0$.

Very recently (c.f. [27]) the authors have obtained a quantitative version of the endpoint estimate for arbitrary weights, namely Theorem 4. This result is analogous to the one obtained by the first author and T. Hytönen for singular integrals in [15].

Theorem 5. Let $T$ be a Calderón-Zygmund operator and $b \in B M O$. If $w \geq 0$ is a weight then, for every $\varepsilon>0$

$$
w\left(\left\{x \in \mathbb{R}^{n}:|[b, T] f(x)|>\lambda\right\}\right) \leq \frac{c}{\varepsilon^{2}} \int_{\mathbb{R}^{n}} \Phi\left(\|b\|_{B M O} \frac{|f|}{\lambda}\right) M_{L(\log L)^{1+\varepsilon} w d x .}
$$

The main novelty here is the appearance of the sharp factor $\frac{1}{\varepsilon^{2}}$ reflecting again the higher singularity of the operator. As a corollary of this result we can derive the following result obtained previously by C. Ortiz-Caraballo in [23],

$$
w\left(\left\{x \in \mathbb{R}^{n}:|[b, T] f(x)|>\lambda\right\}\right) \leq C \Phi\left([w]_{A_{1}}\right)^{2} \int_{\mathbb{R}^{n}} \Phi\left(\|b\|_{B M O} \frac{|f|}{\lambda}\right) w d x .
$$

We remark that it seems that the conjugation method cannot be applied to prove this estimate. Therefore, estimate (5) or Theorem 4 works, so far, for Calderón-Zygmund operators not for general linear operators assuming a minimal appropriate weighted weak type estimate. 
Another interesting difference between Calderón-Zygmund operators and commutators concerns their local behavior. A very nice way of expressing this is by means of the following estimate due to Karagulyan [18]: there exists a constant $c>0$ such that for each cube $Q$ and for each function $f$ supported on the cube $Q$

$$
\frac{1}{|Q|}|\{x \in Q:|T f(x)|>t M f(x)\}| \leq c e^{-c t} \quad t>0
$$

This result can be seen as an improvement of Buckley's exponential decay theorem [3] which is a very useful result. For instance, it allows to improve in a quantitative way the classical good- $\lambda$ inequality between $T$ and $M$ : if $p \in(0, \infty)$ and $w \in A_{\infty}$

$$
\|T f\|_{L^{p}(w)} \leq c_{T} p[w]_{A_{\infty}}\|M(f)\|_{L^{p}(w)} .
$$

Motivated by this result of Karagulyan, Ortiz-Caraballo, Rela and the first author developed a new method for proving (6) in [24]. This method is flexible enough to deal with other operators including the commutators. In particular, we have the following sub-gaussian estimate.

Theorem 6. Let $T$ be a Calderón-Zygmund operator and $b \in B M O$, then there exists a constant $c>0$ such that for each $f$

$$
\sup _{Q} \frac{1}{|Q|}\left|\left\{x \in Q:\left|[b, T]\left(f \chi_{Q}\right)(x)\right|>t M^{2} f(x)\right\}\right| \leq c e^{-\sqrt{c t|| b \|_{B M O}}} \quad t>0 .
$$

We will show in Section 2 that this subexponential decay is fully sharp. In Section 3 , we will provide a new proof of (6) based on the pointwise domination: if $T$ is a Calderón-Zygmund operator, then it is possible to find a finite set of $\eta$-sparse families $\left\{\mathcal{S}_{j}\right\}_{j=1}^{3^{n}}$ (see Section 3 for the definitions) contained in the same or in different dyadic lattices $\mathcal{D}_{j}$ and depending on $f$ such that

$$
|T f(x)| \leq c_{T} \sum_{j=1}^{3^{n}} A_{\mathcal{S}_{j}} f(x)
$$

where

$$
A_{\mathcal{S}_{j}} f(x)=\sum_{Q \in \mathcal{S}_{j}} \frac{1}{|Q|} \int_{Q}|f| \chi_{Q}(x) .
$$

See Section 3 for details, in particular Theorem 7.

In view of the interest of an estimate like (8) it would be relevant to produce a counterpart for commutators. The "natural" sparse operator for these commutators would be

$$
B_{\mathcal{S}} f(x)=\sum_{Q \in \mathcal{S}}\|f\|_{L \log L, Q} \chi_{Q}(x) .
$$

The reason that leads to consider this sparse operator in terms of the average $\|\cdot\|_{L \log L, Q}$ is due to the intimate relationship of commutators and $M^{2}$ which is an operator pointwise equivalent to $M_{L \log L}$. In Section 3 we prove the impossibility of having a domination theorem for commutators by these "sparse" operators. 


\section{First observation: Sharpness of the subexponential local DeCAY}

We prove in this section that Theorem 6 is sharp, i.e., we can find a CalderónZygmund operator $T$, a symbol $b \in B M O$ a function $f$ and a cube $Q$ such that

$$
\frac{1}{|Q|}\left|\left\{x \in Q:|[b, T] f(x)|>t M^{2} f(x)\right\}\right| \geq c e^{-\sqrt{c t\|b\|_{B M O}}}
$$

for some constant $c>0$. More precisely we have the following.

Observation 1. Let $b(x)=\log |x|$, then we can find a constant $c>0$ such that

$$
\left|\left\{x \in(0,1):\left|[b, H]\left(\chi_{(0,1)}\right)(x)\right|>t\right\}\right| \geq e^{-\sqrt{c t}}
$$

where $H$ stands for the Hilbert transform.

Proof. Let $f(x)=\chi_{(0,1)}(x)$. We are going to show that

$\left|\left\{x \in(0,1):|[b, H] f(x)|>t M^{2} f(x)\right\}\right|=|\{x \in(0,1):|[b, H] f(x)|>t\}| \geq c e^{-\sqrt{\alpha t}} \quad t>0$.

For $x \in(0,1)$ we have that

$$
[b, H] f(x)=\int_{0}^{1} \frac{\log (x)-\log (y)}{x-y} d y=\int_{0}^{1} \frac{\log \left(\frac{x}{y}\right)}{x-y} d y=\int_{0}^{1 / x} \frac{\log \left(\frac{1}{t}\right)}{1-t} d t .
$$

Now we observe that

$$
\int_{0}^{1 / x} \frac{\log \left(\frac{1}{t}\right)}{1-t} d t=\int_{0}^{1} \frac{\log \left(\frac{1}{t}\right)}{1-t} d t+\int_{1}^{1 / x} \frac{\log \left(\frac{1}{t}\right)}{1-t} d t
$$

and since $\frac{\log \left(\frac{1}{t}\right)}{1-t}$ is positive for $(0,1) \cup(1, \infty)$ we have for $0<x<1$ that

$$
|[b, H] f(x)|>\int_{1}^{1 / x} \frac{\log \left(\frac{1}{t}\right)}{1-t} d t .
$$

Finally, a computation shows that

$$
\int_{1}^{1 / x} \frac{\log \left(\frac{1}{t}\right)}{1-t} d t \approx\left(\log \frac{1}{x}\right)^{2} \quad x \rightarrow 0 .
$$

Consequently, we have that for some $x_{0}<1$

$$
|[b, H] f(x)|>c\left(\log \frac{1}{x}\right)^{2} \quad 0<x<x_{0} .
$$

and then for some $t_{0}>0$,

$$
|\{x \in(0,1):|[b, H] f(x)|>t\}| \geq\left|\left\{x \in\left(0, x_{0}\right): c\left(\log \frac{1}{x}\right)^{2}>t\right\}\right|=e^{-\sqrt{t / c}} \quad t>t_{0}
$$

as we wanted to prove. 


\section{SECOnd ObSERVATiOn: A "NATURAL" BUt FAlSE SPARSE DOMinAtion ReSUlt FOR COMMUTATORS}

Before stating the result we are going to prove in this section we need some notation. We borrow it from [21].

Definition 1 (Dyadic child). Let $Q$ be a cube (with sides parallel to the axis). We call dyadic child any of the $2^{n}$ cubes obtained by partitioning $Q$ by $n$ "median hyperplanes" (planes parallel to the faces of $Q$ and dividing each edge into 2 equal parts).

If we iterate the partition process of the preceding definition we obtain a standard dyadic lattice $\mathcal{D}(Q)$ of subcubes of $Q$ which has the usual properties:

(1) For each $k=0,1,2, \ldots$ cubes in the $k$-th generation have sidelength $2^{-k}$ and tile $Q$ in a regular way.

(2) Each $Q^{\prime}$ in the $k$-th generation has $2^{n}$ children in the in the $(k+1)$-th generation contained in it and one and only one parent in the $(k-1)$-th generation containing it (unless it is $Q$ itself).

(3) If $Q^{\prime}, Q^{\prime \prime} \in \mathcal{D}(Q)$, then $Q^{\prime} \cap Q^{\prime \prime}=\emptyset$ or $Q^{\prime} \subseteq Q^{\prime \prime}$ or $Q^{\prime \prime} \subseteq Q^{\prime}$.

(4) If $Q^{\prime} \in \mathcal{D}(Q)$, then $\mathcal{D}\left(Q^{\prime}\right) \subseteq \mathcal{D}(Q)$.

Definition 2 (Dyadic lattice). A dyadic lattice $\mathcal{D}$ in $\mathbb{R}^{n}$ is any collection of cubes such that

(DL-1) If $Q \in \mathcal{D}$ then each dyadic child of $Q$ is in $\mathcal{D}$ as well.

$(\mathrm{DL}-2) \quad$ If $Q^{\prime}, Q^{\prime \prime} \in \mathcal{D}$ there exists $Q \in \mathcal{D}$ such that $Q^{\prime}, Q^{\prime \prime} \in \mathcal{D}(Q)$.

(DL-3) If $K$ is a compact set of $\mathbb{R}^{n}$ there exists $Q \in \mathcal{D}$ such that $K \subseteq Q$.

There is an easy way to build a dyadic lattice by considering a increasing sequence of dyadic cubes $Q_{j}$ such that $\cup_{j=1}^{\infty} Q_{j}=\mathbb{R}^{n}$. Then

$$
\mathcal{D}=\bigcup_{j=1}^{\infty} \mathcal{D}\left(Q_{j}\right)
$$

is a dyadic lattice.

Definition 3. Let $\eta \in(0,1)$. We say that a family of cubes $\mathcal{S} \subseteq \mathcal{D}$ is $\eta$-sparse if for each $Q \in \mathcal{S}$ we can find a measurable subset $E(Q) \subset Q$ such that:

(1) $E(Q)$ 's are pairwise disjoint.

(2) $\eta|Q| \leq|E(Q)|$

Definition 4. Let $\Lambda>1$. We say a family of cubes $\mathcal{S}$ is $\Lambda$-Carleson if for every cube $Q \in \mathcal{D}$ we have

$$
\sum_{P \in \mathcal{S}, P \subset Q}|P| \leq \Lambda|Q| .
$$

There is an interesting relation between Carleson and sparse families that we summarize in the following lemma

Lemma 1. If $\mathcal{S}$ is a $\Lambda$-Carleson family of cubes then it is $\frac{1}{\Lambda}$-sparse. Conversely if $\mathcal{S}$ is a $\eta$-sparse family of cubes then it is a $\frac{1}{\eta}$-Carleson family of cubes. 
Armed with all these definitions we can state the following pointwise domination theorem.

Theorem 7. Let $T$ be a Calderón-Zygmund operator. There is a finite set of $\eta$-sparse families $\left\{\mathcal{S}_{j}\right\}_{j=1}^{3^{n}}$ contained in the same or in different dyadic lattices $\mathcal{D}_{j}$ and depending on $f$ such that

$$
T^{*} f(x) \leq c_{T, n} \sum_{j=1}^{3^{n}} A_{\mathcal{S}_{j}} f(x)
$$

where $A_{\mathcal{S}_{j}} f(x)=\sum_{Q \in \mathcal{S}_{j}} \frac{1}{|Q|} \int_{Q}|f| \chi_{Q}(x)$.

The proof of this result can be found in [21] and [7]. In [19] M. Lacey obtains the same estimate for Calderón-Zygmund operators that satisfy a Dini condition. Recently a fully quantitative version of Lacey's result was obtained in [16] and even more recently this quantitative version has been simplified in [20].

As a sample of the interest of this result we give a different proof of the exponential estimate (6): there exists a constant $c>0$ such that for each cube $Q$ and for each $f$ supported on the cube $Q$

$$
\frac{1}{|Q|}\left|\left\{x \in Q: T^{*} f(x)>t M f(x)\right\}\right| \leq c e^{-c t} \quad t>0,
$$

To prove this result we will use the classical vector-valued extension of the maximal function introduced by Fefferman and Stein in [11] that can be written as follows:

$$
\bar{M}_{q} f(x)=\left(\sum_{j=1}^{\infty}\left(M f_{j}(x)\right)^{q}\right)^{1 / q}=|M f(x)|_{q}
$$

where $f=\left\{f_{j}\right\}_{j=1}^{\infty}$ is a vector-valued function.

Indeed, consider the distribution set

$$
S_{Q}:=\left\{x \in Q: T^{*} f(x)>t M f(x)\right\} .
$$

Then, by Theorem 7 we have that

$$
\begin{aligned}
\left|S_{Q}\right| & \leq\left|\left\{x \in Q: c_{T, n} \sum_{j=1}^{3^{n}} A_{\mathcal{S}_{j}} f(x)>t M f(x)\right\}\right| \\
& \leq \sum_{j=1}^{3^{n}}\left|\left\{x \in Q: A_{\mathcal{S}_{j}} f(x)>t\left(3^{n} c_{T, n}\right)^{-1} M f(x)\right\}\right| \\
& \leq \sum_{j=1}^{3^{n}}\left|\left\{x \in Q: \sum_{Q \in \mathcal{S}_{j}} \chi_{Q}(x)>t\left(3^{n} c_{T, n}\right)^{-1}\right\}\right|
\end{aligned}
$$

since trivially,

$$
A_{\mathcal{S}_{j}} f(x) \leq M f(x) \sum_{Q \in \mathcal{S}_{j}} \chi_{Q}(x)
$$


We now use one of the key estimates from [24]. Indeed, let $\{E(Q)\}_{Q \in \mathcal{S}_{j}}$ be the family of sets from Definition 3. We have then for some $c>0$

$$
\begin{aligned}
\sum_{Q \in \mathcal{S}_{j}} \chi_{Q}(x) & =\sum_{Q \in \mathcal{S}_{j}}\left(\frac{1}{|Q|}|Q|\right)^{q} \chi_{Q}(x) \\
& \leq c \sum_{Q \in \mathcal{S}_{j}}\left(\frac{1}{|Q|}|E(Q)|\right)^{q} \chi_{Q}(x) \\
& \leq c \sum_{Q \in \mathcal{S}_{j}}\left(\frac{1}{|Q|} \int_{Q} \chi_{E(Q)}(y) d y\right)^{q} \chi_{Q}(x) \\
& \leq c\left(\bar{M}_{q}\left(\left\{\chi_{E(Q)}\right\}_{Q \in \mathcal{S}_{j}}\right)(x)\right)^{q} \\
& \leq c\left(\bar{M}_{q} g_{j}(x)\right)^{q},
\end{aligned}
$$

where $g_{j}=\left\{\chi_{E(Q)}\right\}_{Q \in \mathcal{S}_{j}}$. Now, since $\{E(Q)\}_{Q \in \mathcal{S}_{j}}$ is a pairwise disjoint family of subsets, we have that for any $j$

$$
\left\|g_{j}(x)\right\|_{\ell^{q}}=\left(\sum_{Q \in \mathcal{S}_{j}}\left(\chi_{E(Q)}(x)\right)^{q}\right)^{1 / q} \leq 1 .
$$

We finish our proof recalling that if $\left|g_{j}\right|_{\ell^{q}} \in L^{\infty}$, then $\left(\bar{M}_{q} g_{j}(x)\right)^{q} \in \operatorname{ExpL}$ (see [11]) from which we conclude the desired inequality (11):

$$
\left|S_{Q}\right| \leq c e^{-c t}|Q|, \quad t>0
$$

Observation 2. Let $T$ be a Calderón-Zygmund operator and $b \in B M O$. It is not possible to find a finite set of $\eta$-sparse families $\left\{\mathcal{S}_{j}\right\}_{j=1}^{N}$, with $N$ dimensional, contained in the same or in different dyadic lattices $\mathcal{D}_{j}$ and depending on $f$ such that

$$
|[b, T] f(x)| \leq c_{b, T} \sum_{j=1}^{N} B_{\mathcal{S}_{j}} f(x) \quad \text { a.e. } x \in \mathbb{R}^{n}
$$

where $B_{\mathcal{S}_{j}} f(x)=\sum_{Q \in \mathcal{S}_{j}}\|f\|_{L \log L, Q} \chi_{Q}(x)$.

We are going to give two proofs of this result. The first one is based on the Rubio de Francia algorithm.

Proof 1. Suppose that equation (13) holds, then we can prove the following $L^{1}$ inequality

$$
\|[b, T] f\|_{L^{1}(w)} \leq c[w]_{A_{1}}\left\|M^{2} f\right\|_{L^{1}(w)} .
$$


Indeed,

$$
\begin{aligned}
\|[b, T] f\|_{L^{1}(w)} & \leq c_{b, T} \sum_{j=1}^{N}\left\|B_{\mathcal{S}_{j}} f\right\|_{L^{1}(w)} \\
& \leq c_{b, T} \sum_{j=1}^{N} \sum_{Q \in \mathcal{S}_{j}}\|f\|_{L \log L, Q} \frac{w(Q)}{|Q|}|Q| \\
& \leq \frac{c_{b, T}}{\eta} \sum_{j=1}^{N} \sum_{Q \in \mathcal{S}_{j}}\|f\|_{L \log L, Q} \frac{w(Q)}{|Q|}|E(Q)| \\
& \leq \frac{c_{b, T}}{\eta} \sum_{j=1}^{N} \sum_{Q \in \mathcal{S}_{j}} \int_{E(Q)} M_{L \log L} f(x) M w(x) d x \\
& \leq N \frac{c_{b, T}}{\eta}[w]_{A_{1}}\left\|M^{2} f\right\|_{L^{1}(w)},
\end{aligned}
$$

since $M^{2} \approx M_{L \log L}$. We claim now the $L^{p}$ version,

$$
\|[b, T] f\|_{L^{p}\left(\mathbb{R}^{n}\right)} \leq c_{n} p\left\|M^{2} f\right\|_{L^{p}\left(\mathbb{R}^{n}\right)} \quad p>1 .
$$

Indeed, by duality we can find $g \geq 0$ in $L^{p^{\prime}}\left(\mathbb{R}^{n}\right)$ with unit norm such that

$$
\|[b, T] f\|_{L^{p}\left(\mathbb{R}^{n}\right)}=\int_{\mathbb{R}^{n}}|[b, T] f(x)| g(x) d x .
$$

We consider the Rubio de Francia algorithm

$$
R g=\sum_{k=0}^{\infty} \frac{M^{k}(g)}{\|M\|_{L^{p^{\prime}\left(\mathbb{R}^{n}\right)}}^{k}} .
$$

It's a straightforward computation that $R(g)$ is an $A_{1}$ weight with constant

$$
[R g]_{A_{1}} \leq 2\|M\|_{L^{p^{\prime}}} \leq c_{n} p
$$

and also that $g \leq R g$ and $\|R g\|_{L^{p^{\prime}}} \leq 2\|g\|_{L^{p^{\prime}\left(\mathbb{R}^{n}\right)}}=2$. Then have that

$$
\int_{\mathbb{R}^{n}}|[b, T] f(x)| g(x) d x \leq \int_{\mathbb{R}^{n}}|[b, T] f(x)| R g(x) d x
$$

and using equation (14) and Hölder inequality

$$
\begin{aligned}
& \int_{\mathbb{R}^{n}}|[b, T] f(x)| R g(x) d x \leq c[R g]_{A_{1}} \int_{\mathbb{R}^{n}} M^{2} f(x) R g(x) d x \\
& \leq c p \int_{\mathbb{R}^{n}} M^{2} f(x) R g(x) d x \leq c p\left\|M^{2} f\right\|_{L^{p}\left(\mathbb{R}^{n}\right)}\|R g\|_{L^{p^{\prime}\left(\mathbb{R}^{n}\right)}} \\
& \leq c p\left\|M^{2} f\right\|_{L^{p}\left(\mathbb{R}^{n}\right)} .
\end{aligned}
$$

Hence equation (15) is established. Now since

$$
\left\|M^{2}\right\|_{L^{p}\left(\mathbb{R}^{n}\right)} \leq c_{n}\left(p^{\prime}\right)^{2} \quad p>1
$$


we have that

$$
\|[b, T]\|_{L^{p}\left(\mathbb{R}^{n}\right)} \leq c p\left(p^{\prime}\right)^{2} \quad p>1
$$

Now let us observe that if we take $[b, H] f$ with $b(x)=\log |x|$ and $f(x)=\chi_{(0,1)}(x)$ then

$$
\|[b, H] f\|_{L^{p}(\mathbb{R})} \geq c p^{2} \quad p>1,
$$

and this leads to a contradiction when $p \rightarrow \infty$. To prove this lower estimate we use estimate (9) from Theorem 1. Indeed, for some $t_{0}>0$

$$
\begin{aligned}
\|[b, H] f\|_{L^{p}(\mathbb{R})} & \geq\|[b, H] f\|_{L^{p, \infty}(\mathbb{R})}=\sup _{t>0} t|\{x \in \mathbb{R}:|[b, H] f(x)|>t\}|^{\frac{1}{p}} \\
& \geq \sup _{t>t_{0}} t\left|\left\{x \in\left(0, x_{0}\right): c\left(\log \frac{1}{x}\right)^{2}>t\right\}\right|^{\frac{1}{p}} \\
& \geq \sup _{t>t_{0}} t c e^{\frac{-\sqrt{t}}{p}} \geq c p^{2} t_{0} e^{-\sqrt{t_{0}}}
\end{aligned}
$$

and this concludes the first proof.

For the second proof we will rely on the sharpness result that was settled in the previous section.

Proof 2. Assume again that equation (13) holds. Then, for some $c>1$

$$
\left|\left\{x \in Q:|[b, T] f(x)|>t M^{2} f(x)\right\}\right| \leq\left|\left\{x \in Q: \sum_{j=1}^{N} \sum_{Q \in \mathcal{S}_{j}}\|f\|_{L \log L, Q} \chi_{Q}(x)>\operatorname{ct} M^{2} f(x)\right\}\right|
$$

We use a similar argument as for the proof of (11). Now, recalling that $M^{2} f \simeq M_{L \log L} f$ this is bounded by

$$
\sum_{j=1}^{N}\left|\left\{x \in Q: \sum_{Q \in \mathcal{S}_{j}} \chi_{Q}(x)>c t\right\}\right|
$$

and we can proceed exactly as in the proof of (11) to derive

$$
\left|\left\{x \in Q: \sum_{Q \in \mathcal{S}} \chi_{Q}(x)>t C\right\}\right| \leq c|Q| e^{-\alpha t} .
$$

Combining we arrive to

$$
\frac{1}{|Q|}\left|\left\{x \in Q:|[b, T] f(x)|>t M^{2} f(x)\right\}\right| \leq c e^{-\alpha t} \quad t>0
$$

which is a contradiction by Observation 1 .

The correct pointwise control for the commutator seems to be the following one

Conjecture 1. Let $T$ be a Calderón-Zygmund operator and $b \in B M O$. Then

$$
|[b, T] f(x)| \leq C(n, T)\|b\|_{B M O} \sum_{i, j=1}^{N} A_{\mathcal{S}_{i}}\left(A_{\mathcal{S}_{j}} f\right)(x)
$$


where $A_{\mathcal{S}_{j}} f(x)=\sum_{Q \in \mathcal{S}_{j}} \frac{1}{|Q|} \int_{Q}|f(y)| d y \chi_{Q}(x)$ and the sparse families $\mathcal{S}_{j}$ are not necessarily subfamilies of the same dyadic lattice.

If this conjecture holds it would be very easy to recover the main theorem from [4] since it suffices to iterate the following estimate:

$$
\left\|A_{\mathcal{S}_{j}} f\right\|_{L^{p}(w)} \leq C_{n, p}[w]_{A_{p}}^{\max \left\{1, \frac{1}{p-1}\right\}}\|f\|_{L^{p}}
$$

which was studied in [9, 10] (see also [21]).

\section{Third observation: The failure of A Endpoint estimate motivated By THE CONJUGATION METHOD}

In this section we consider the following family of operators:

$$
f \rightarrow T_{w}(f):=w T\left(\frac{f}{w}\right)
$$

where $w$ is a weight and $T$ is a Calderón-Zygmund operator. We already mentioned in the introduction that these operators are of interest since they are very much related to commutators due to the conjugation method. We emphasized that the case $w \in A_{1}$ is special since $T_{w}$ is of weak type $(1,1)$ as a consequence of the main results from [8]. Understanding the case $w \in A_{p}$ would be more interesting due to its connection with the conjugation method. However, $T_{w}$ is not of weak type $(1,1)$ in general since there are weights $w \in A_{p} \backslash A_{1}$ for which

$$
\left\|T_{w}\right\|_{L^{1}\left(\mathbb{R}^{n}\right) \rightarrow L^{1, \infty}\left(\mathbb{R}^{n}\right)}=\infty
$$

being the purpose of this section to show the existence of such weights. In fact we are going to show something worst replacing $T$ by the less singular operator $M$.

Observation 3. Let $1<p<\infty$, then there is $w \in A_{p} \backslash A_{1}$ such that

$$
\left\|M_{w}\right\|_{L^{1}\left(\mathbb{R}^{n}\right) \rightarrow L^{1, \infty}\left(\mathbb{R}^{n}\right)}=\infty \text {. }
$$

Proof. In dimension 1 we choose the $A_{p}$ weight $w(x)=|x|^{-\delta(1-p)}$ with $\delta \in\left(0, \min \left\{1, \frac{1}{p-1}\right\}\right)$ and $f=\chi_{[0,1]}$ so that $f \in L^{1}(w)$. We prove that

$$
\left\|w M\left(\frac{f}{w}\right)\right\|_{L^{1, \infty}(\mathbb{R})}=\infty .
$$

Indeed, a computation shows that for $x>1$

$$
M\left(\frac{\chi_{(0,1)}}{w}\right)(x) \geq \frac{1}{x} \frac{1}{\beta}
$$

with $\beta=1+\delta(1-p)$ and then

$$
\left\|w M\left(\frac{f}{w}\right)\right\|_{L^{1, \infty}(\mathbb{R})} \geq \frac{1}{\beta} \sup _{t>0} t\left|\left\{x>1: x^{-\delta(1-p)-1}>t\right\}\right|=\frac{1}{\beta} \sup _{1>t>0} t\left(\left(\frac{1}{t}\right)^{\frac{1}{\beta}}-1\right)=\infty
$$

since $\beta \in(0,1)$. 
An interesting question is to find a necessary and sufficient condition for the boundedness of this operator, namely, characterize the weights $w$ for which

$$
\left\|M_{w}\right\|_{L^{1}\left(\mathbb{R}^{n}\right) \rightarrow L^{1, \infty}\left(\mathbb{R}^{n}\right)}<\infty .
$$

In [22] Muckenhoupt and Wheeden proved that this inequality holds for $w \in A_{1}$ in the real line and also obtained a necessary condition on the weights, namely

$$
\left\|\frac{w \chi_{Q}}{|\cdot-x|^{n}}\right\|_{L^{1, \infty}\left(\mathbb{R}^{n}\right)} \leq c w(x) \quad \text { a.e. } x \in \mathbb{R}^{n}
$$

but we don't know whether is sufficient or not.

To end this section we show that can go further and prove a negative result for possible $L \log L$ type estimates.

Observation 4. Let $1<p<\infty$, and let $\Phi(t)=t \log (e+t)^{\alpha}, \alpha>0$. Then we can find $w \in A_{p} \backslash A_{1}$ and $f$ such that there's no $c>0$ for which

$$
\left|\left\{x \in \mathbb{R}^{n}: w M\left(\frac{f}{w}\right)>t\right\}\right| \leq c \int_{\mathbb{R}^{n}} \Phi\left(\frac{|f(x)|}{t}\right) d x .
$$

Proof. As above we do it for the case $n=1$. We assume the contrary, namely there is a finite constant $c>0$ such that (18) holds for any nonnegative $f$. Let $f=\chi_{(0,1)}$. For this choice of $f$ the right hand side of (18) equals $\Phi\left(\frac{1}{t}\right)$ and we have that

$$
\sup _{t>0} \frac{1}{\Phi\left(\frac{1}{t}\right)}\left|\left\{x \in \mathbb{R}: w M\left(\frac{\chi_{(0,1)}}{w}\right)>t\right\}\right|<\infty .
$$

Choose again the $A_{p}$ weight $w(x)=|x|^{-\delta(1-p)}$ with $\delta \in\left(0, \min \left\{1, \frac{1}{p-1}\right\}\right)$. Proceeding and using the same notation as in the proof of Observation 3 we have that

$$
\begin{aligned}
\sup _{t>0} \frac{1}{\Phi\left(\frac{1}{t}\right)}\left|\left\{x \in \mathbb{R}: w M\left(\frac{\chi(0,1)}{w}\right)>t\right\}\right| & \geq c \sup _{0<t<1} \frac{1}{\Phi\left(\frac{1}{t}\right)}\left[\left(\frac{1}{t}\right)^{\frac{1}{\beta}}-1\right] \\
& =c \sup _{0<t<1} \frac{t}{\log \left(e+\frac{1}{t}\right)^{\alpha}}\left[\left(\frac{1}{t}\right)^{\frac{1}{\beta}}-1\right]=\infty .
\end{aligned}
$$

since $\beta \in(0,1)$.

\section{REFERENCES}

[1] J. Álvarez, R. J. Bagby, D. S. Kurtz, and C. Pérez. Weighted estimates for commutators of linear operators. Studia Math., 104(2):195-209, 1993.

[2] S. Bloom. A commutator theorem and weighted BMO. Trans. Amer. Math. Soc., 292(1):103-122, 1985.

[3] S. M. Buckley. Estimates for operator norms on weighted spaces and reverse Jensen inequalities. Trans. Amer. Math. Soc., 340(1):253-272, 1993.

[4] D. Chung, M. C. Pereyra, and C. Perez. Sharp bounds for general commutators on weighted Lebesgue spaces. Trans. Amer. Math. Soc., 364(3):1163-1177, 2012.

[5] R. R. Coifman and Y. Meyer. On commutators of singular integrals and bilinear singular integrals. Trans. Amer. Math. Soc., 212:315-331, 1975. 
[6] R. R. Coifman, R. Rochberg, and G. Weiss. Factorization theorems for Hardy spaces in several variables. Ann. of Math. (2), 103(3):611-635, 1976.

[7] J. M. Conde-Alonso and G. Rey. A pointwise estimate for positive dyadic shifts and some applications. ArXiv e-prints, September 2014.

[8] D. Cruz-Uribe, J. M. Martell, and C. Pérez. Weighted weak-type inequalities and a conjecture of Sawyer. Int. Math. Res. Not., (30):1849-1871, 2005.

[9] D. Cruz-Uribe, J. M. Martell, and C. Pérez. Sharp weighted estimates for approximating dyadic operators. Electron. Res. Announc. Math. Sci., 17:12-19, 2010.

[10] D. Cruz-Uribe, J. M. Martell, and C. Pérez. Sharp weighted estimates for classical operators. Adv. Math., 229(1):408-441, 2012.

[11] C. Fefferman and E. M. Stein. Some maximal inequalities. Amer. J. Math., 93:107-115, 1971.

[12] J. García-Cuerva, E. Harboure, C. Segovia, and J. L. Torrea. Weighted norm inequalities for commutators of strongly singular integrals. Indiana Univ. Math. J., 40(4):1397-1420, 1991.

[13] I. Holmes, M. T. Lacey, and B. D. Wick. Commutators in the Two-Weight Setting. ArXiv e-prints, June 2015.

[14] I. Holmes and B. D. Wick. Two Weight Inequalities for Iterated Commutators with Calder \'onZygmund Operators. ArXiv e-prints, September 2015.

[15] T. Hytönen and C. Pérez. Sharp weighted bounds involving $A_{\infty}$. Anal. PDE, 6(4):777-818, 2013.

[16] T. P. Hytönen, L. Roncal, and O. Tapiola. Quantitative weighted estimates for rough homogeneous singular integrals. ArXiv e-prints, October 2015.

[17] S. Janson. Mean oscillation and commutators of singular integral operators. Ark. Mat., 16(2):263$270,1978$.

[18] G. A. Karagulyan. Exponential estimates for the Calderón-Zygmund operator and related problems of Fourier series. Mat. Zametki, 71(3):398-411, 2002.

[19] M. T. Lacey. An elementary proof of the A_2 Bound. ArXiv e-prints, January 2015.

[20] A. K. Lerner. On pointwise estimates involving sparse operators. ArXiv e-prints, December 2015.

[21] A. K. Lerner and F. Nazarov. Intuitive dyadic calculus: the basics. ArXiv e-prints, August 2015.

[22] B. Muckenhoupt and R. L. Wheeden. Some weighted weak-type inequalities for the HardyLittlewood maximal function and the Hilbert transform. Indiana Univ. Math. J., 26(5):801-816, 1977.

[23] C. Ortiz-Caraballo. Quadratic $A_{1}$ bounds for commutators of singular integrals with BMO functions. Indiana Univ. Math. J., 60(6):2107-2129, 2011.

[24] C. Ortiz-Caraballo, C. Pérez, and E. Rela. Exponential decay estimates for singular integral operators. Math. Ann., 357(4):1217-1243, 2013.

[25] C. Pérez. Endpoint estimates for commutators of singular integral operators. J. Funct. Anal., 128(1):163-185, 1995.

[26] C. Pérez and G. Pradolini. Sharp weighted endpoint estimates for commutators of singular integrals. Michigan Math. J., 49(1):23-37, 2001.

[27] C. Pérez and I. P. Rivera-Ríos. Borderline weighted estimates for commutators of singular integrals. ArXiv e-prints, July 2015.

[28] A. Torchinsky. Real-variable methods in harmonic analysis, volume 123 of Pure and Applied Mathematics. Academic Press, Inc., Orlando, FL, 1986.

Carlos Pérez, Department of Mathematics, University of the Basque Country UPV/EhU, IKerbasque, Basque Foundation for Science, Bilbao and BCAM, Basque Center for Applied Mathematics, Bilbao, Spain.

E-mail address: carlos.perezmo@ehu.es

Israel P. Rivera-Ríos, imus \& Departamento de Análisis Matemático, Universidad De Sevilla, Sevilla, Spain

E-mail address: petnapet@gmail.com 